\title{
POLAR: the first dedicated gamma-ray burst polarization experiment
}

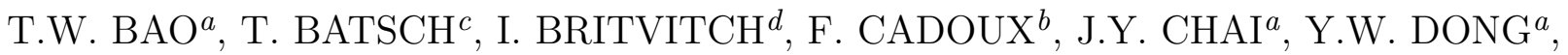 \\ N. GAUVIN ${ }^{e}$, W. HAJDAS ${ }^{d}$, M.N. KONG ${ }^{a}$, C. LECHANOINE-LELUC ${ }^{b}$, Lu LI $^{a}$, J.T. LIU ${ }^{a}$,

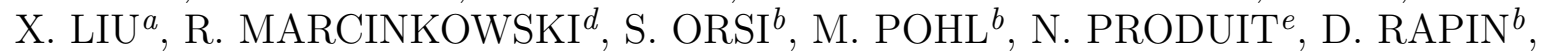 \\ A. RUTCZYNSKA ${ }^{c}$, D. RYBKA ${ }^{d, c}$, H.L. SHI ${ }^{a}$, J.C. SUN ${ }^{a}$, J. SZABELSKI ${ }^{c}$, R.J. WANG ${ }^{a}$, \\ X. $\mathrm{WEN}^{a}$, B.B. $\mathrm{WU}^{a}$, H.L. XIAO ${ }^{a}$, H.H. XU ${ }^{a}$, LI ZHANG ${ }^{a}$, L.Y. ZHANG ${ }^{a}$, S.N. ZHANG ${ }^{a}$, \\ Y.J. ZHANG ${ }^{a}$, A. ZWOLINSKA ${ }^{c}$ \\ ${ }^{a}$ Key Laboratory of Particle Astrophysics, Institute of High Energy Physics, Chinese \\ Academy of Sciences, Beijing, China, 100049; \\ ${ }^{b}$ University of Geneva (DPNC), quai Ernest-Ansermet 24, 1205 Geneva, Switzerland; \\ and Center for Astroparticle Physics, Geneva, Switzerland; \\ ${ }^{c}$ National Centre for Nuclear Research ul. A. Soltana 7, 05-400 Otwock, Swierk, Poland; \\ ${ }^{d}$ Paul Scherrer Institut 5232 Villigen PSI, Switzerland; \\ ${ }^{e}$ University of Geneva, ISDC Data center for Astrophysics, 16, Chemin d'Ecogia, 1290 Versoix \\ Switzerland;
}

\begin{abstract}
POLAR is a Gamma-Ray Burst (GRB) polarization experiment in the energy range 50-500 keV. Detection principle of the gamma-ray polarization is based on the anisotropy of the Compton scattering. POLAR consists of 1600 low-Z plastic scintillator bars, read out by 25 flat-panel multianode photomultipliers. Simulations and experiments have shown that the polarization degree and angle can be retrieved from the modulation curves with the required accuracy. POLAR can reach a minimum detectable polarization of about $10 \%$ (3-sigma level) for several strongest GRB detections per year. Construction and assembly of the Qualification Model (QM) are ongoing, in view of a flight onboard of the Chinese Spacelab TG-2 scheduled for 2014.
\end{abstract}

Keywords: X-ray polarization; Gamma-Ray Burst (GRB), Scintillator detector, Synchrotron radiation, POLAR

\section{INTRODUCTION}

The nature of cosmic gamma-ray bursts (GRBs) as extremely explosive releases of electromagnetic energy has been the focus of astrophysicists attention since soon after their discovery in 1967-1973 by U.S. Vela satellites. ${ }^{1}$ The genuine character of the GRB sources and the energy release mechanisms still remain a mystery and there are several competing theoretical models and mechanisms trying to explain it. Two widely accepted models have different polarization predictions for promptly ejected photons. Therefore, the precise polarization measurements can unveil the mystery of GRBs real nature. In this decade, with the improvement of the technology, there are more and more experimental instruments, which have polarimetry ability. ${ }^{2}$

The POLAR instrument will investigate the physics of photon emission and the nature of energy release GRBs. Highly precise, continuous observations from an unprecedented number of GRBs will be performed to determine the degree of linear polarization at photon energies from about $50 \mathrm{keV}$ to $500 \mathrm{keV}$.

The POLAR detector will for the first time provide unambiguous measurements of the hard X-ray polarization from the prompt emission in GRBs. For several bursts per year the experimental precision will reach a one-sigma

Further author information: (Send correspondence to I.Britvitch)

I.Britvitch.: E-mail: Ilia.Britvitch@psi.ch, Telephone: 41563105685

Space Telescopes and Instrumentation 2012: Ultraviolet to Gamma Ray,

edited by Tadayuki Takahashi, Stephen S. Murray, Jan-Willem A. den Herder

Proc. of SPIE Vol. 8443, 84434Q - @ 2012 SPIE · CCC code: 0277-786/12/\$18 - doi: 10.1117/12.925635

Proc. of SPIE Vol. $844384434 \mathrm{Q}-1$

Downloaded From: https://www.spiedigitallibrary.org/conference-proceedings-of-spie on 19 Apr 2019 Terms of Use: https://www.spiedigitallibrary.org/terms-of-use 
level of about $3 \%$ when averaged over several measurements. As the polarimeter it will be able to observe more than $30 \%$ of the sky offering in addition a large active area $\left(A=250 \mathrm{~cm}^{2}\right)$ and modulation factor $\left(\mu_{100 \%}=0.30\right)$. Given its pointing stability, the instrument is also able to accurately determine the polarization in hard X-rays from the Crab nebula, from other Supernova remnants as well as from several Soft Gamma Repeaters. The POLAR instrument will be placed on board the second Chinese Space Station Tian-Gong (Heavenly Palace).

In December 2010, the Chinese General Establishment of Space Science and Application (GESSA) reviewed the POLAR mission, corresponding to a Critical Design Review according to ESA standards. POLAR passed the review on December 9, 2010. An EM review was held in June 2011 and passed successfully. With this, the POLAR team received the go-ahead to enter into phase (design and development phase) with two qualification models to be delivered in 3rd quarter of 2012, and a flight model in 2nd quarter of 2013. The TG-2 launch is foreseen no earlier than middle of 2014.

\section{POLAR DETECTOR}

The POLAR polarimeter is a space-borne compact detector based on the principle of Compton scattering and is dedicated to the polarization measurement of GRB photons. ${ }^{3}$ The azimuthal distribution of scattered photons has an unique relationship with the direction, energy and linear polarization level of the incident photons.

The main part of the POLAR mechanical design is its scintillator target. It is composed of 1600 plastic scintillator bars wrapped in a highly reflective foil. The target is divided into 25 modules each consisting of 64 bars $\left(6 \times 6 \times 176 \mathrm{~mm}^{3}\right)$. Each unit contains a flat panel multi anode photomultiplier tube (MAPMT; H8500, Hamamatsu), mechanically coupled to the bottoms of the scintillator bars via a $0.7 \mathrm{~mm}$ thick transparent optical pad. The whole structure is enclosed in a $1 \mathrm{~mm}$ thick carbon fiber socket that ensures mechanical stability.

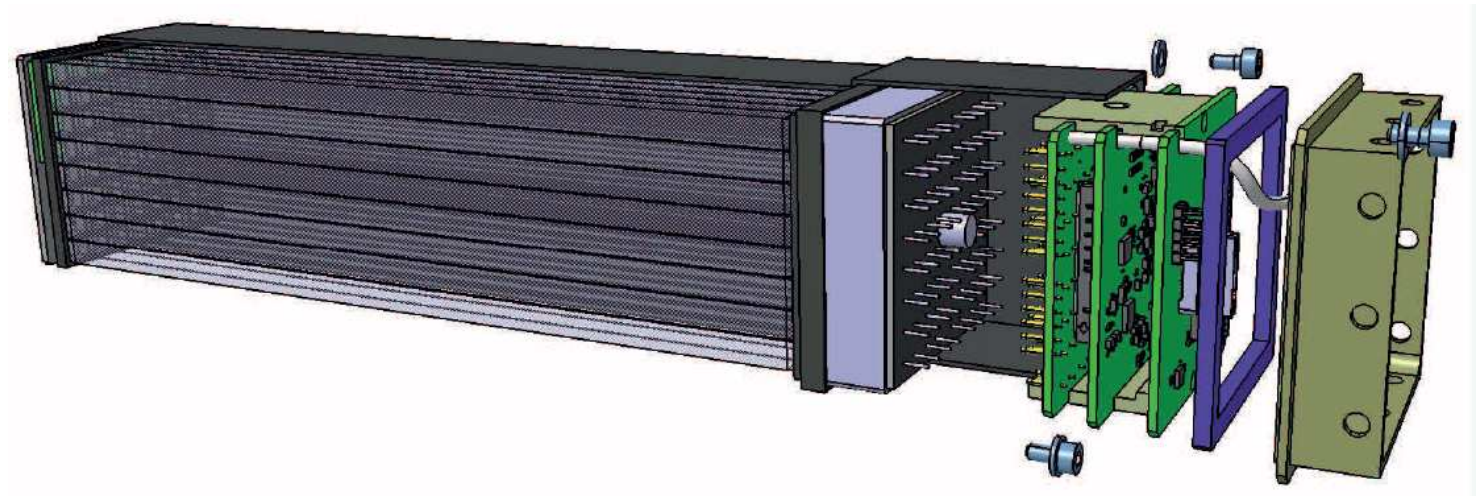

Figure 1. Exploded view of one POLAR modular unit(plastic bars, FEE, MAPMT, mechanical frame).

Electrical signals coming from the MAPMT are first processed by an ASIC and FPGA at the frontend electronics (FEE), then sent to a central processing trigger unit(CT), where the trigger decision is taken based on analysis of digital outputs of all FEE modules. This modular design provides a good mechanical,thermal and electrical features. It also facilitates the interchange of modules during the testing phase of the detector. The bars in each modular unit are kept together with two aligning plastic frames located at the top and at the bottom of the carbon fiber sockets to provide resistance to vibrations and to reduce the optical crosstalk between adjacent channels. Figure 1 shows an exploded view of a modular unit.

All 25 modules are kept in place with an aligning frame located at the bottom of the carbon fiber sockets. The overall volume of the target enclosed in a $3 \mathrm{~mm}$ thick carbon fiber box that enhances the mechanical stability and acts as a shield against the low energy charged particles. Total size of the detector is $45 \mathrm{x} 45 \times 25 \mathrm{~cm}^{3}$, the mass $\approx 30 \mathrm{~kg}$, and its power consumption below $50 \mathrm{~W}$. The detector is located on the outside of the spacecraft facing deep space. Additional subsystems: central trigger, low/high voltages power supplies are also located outside of the spacecraft constituting so called OBOX (See Fig. 2). Only the central computer of POLAR is located inside of the spacecraft (IBOX). 


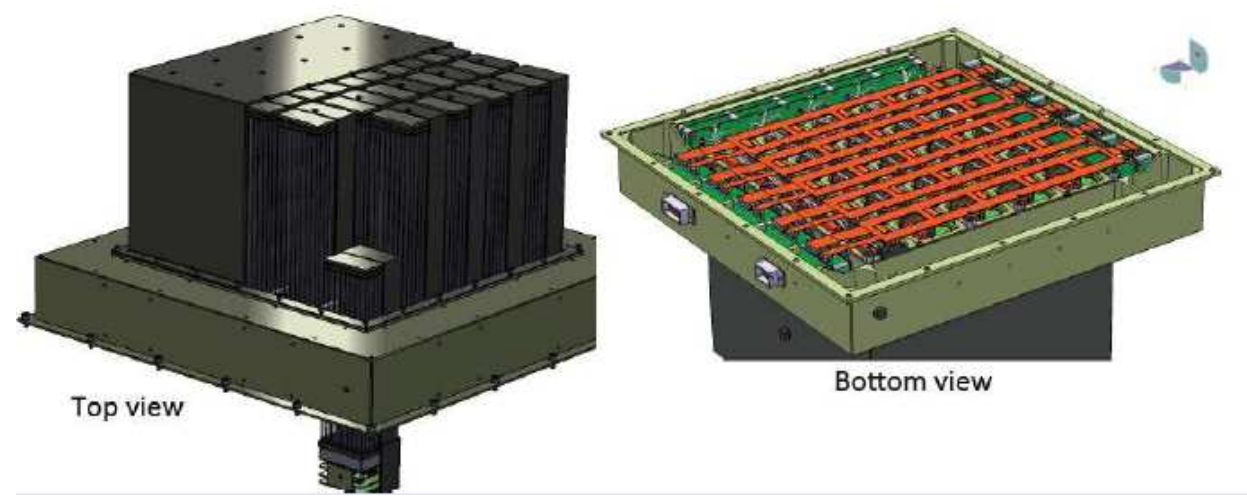

Figure 2. Scheme of POLAR OBOX.

\subsection{The scintillation element}

Plastic bars used to construct the POLAR scintillator target are made out of the EJ-248M (Eljen Technology ${ }^{4}$ ) scintillating material. The plastic scintillator material has been chosen because of its fast response and its low atomic number. Both favor Compton scattering as the interaction process for the hard X-ray photons. The maximum wavelength emission of EJ-248M is $410 \mathrm{~nm}$ with a light yield of about 1 photon per $90 \mathrm{eV}$. It provided a base for dimensioning of POLAR instrument with respect to the measurements accuracy and photon statistics. The scintillator bars were manufactured in the shape of cuboid with a short, square prism at one end. The envelope dimensions are $6 \times 6 \times 176 \mathrm{~mm}^{3}$. The width of the bar fits the typical ranges of recoil electrons and matches the pitch of the selected MAPMT $(6.08 \mathrm{~mm})$. To reduce the cross-talk it was decided to decrease the cross-section of the bottom end of the scintillator bars, shaping that part as a truncated square pyramid (See Fig. 3).

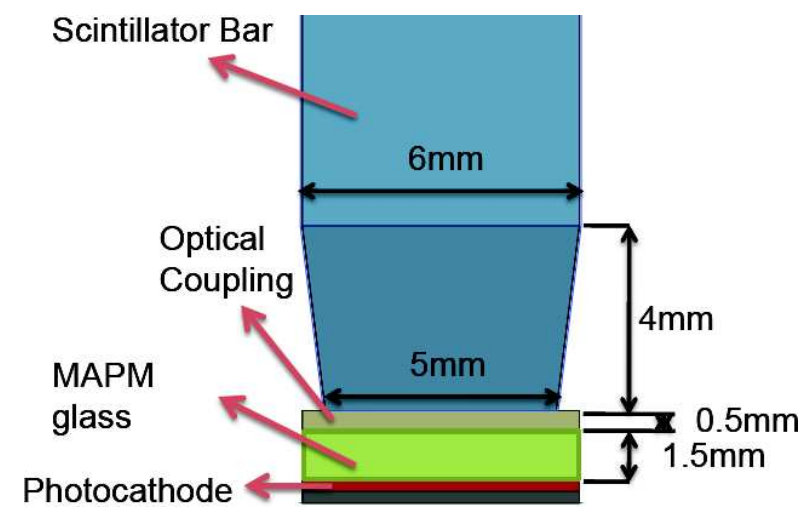

Figure 3. Interfacing of MAPMT with the plastic scintillator. One can see the bottom of the scintillator bar shaped as a truncated square pyramid. The corresponding optical coupling, MAPMT photocathode and glass window are also represented. ${ }^{5}$

The whole length of each single plastic scintillator bar from the POLAR target is enveloped by an enhanced specular reflector (Vikuiti Enhanced Specular Reflector (ESR), $3 \mathrm{M}^{6}$ ) of $65 \mu \mathrm{m}$ thickness. The procedure ensures keeping a thin air or vacuum gap between the foil and the scintillator surface. Such the gap guarantees total reflexion for all angles below $68^{\circ}$ due to a lower refractive index. The wrapping contributes by reflecting back into the scintillator the majority of the optical photons that had been refracted.

\subsection{Data readout system}

The data acquisition in POLAR experiment consists of three stages of processing: Frontend Electronics, Central Trigger, Central Computer. The Frontend modules are responsible for the signal readout from the plastic 
scintillator detectors and for the implementation of the simple local trigger functionality at the module level. At the same time the Frontend communicates with the Central Trigger providing to it information about digital pattern from the detected event. This way it participates in the multi-module triggering scheme. Besides that the Central Trigger is also responsible for full, digital/analog readout of all 25 Frontend modules and reconstruction of the physical events including timing of the experiment. The full event data packet is then sent, with usage of the Central Computer, to the spacecraft for storage and future transmission to Earth.

\subsubsection{The Frontend Electronics Module}

The basic unit of POLAR, the Frontend module, is the ensemble of electronic components distributed over three PCBs boards. The POLAR FEE consists of the following components: high voltage divider and RC coupling board (HVRC PCB), analog signal readout and preprocessing board (ASIC PCB) and Interface board (IF PCB) (See Fig. 4).

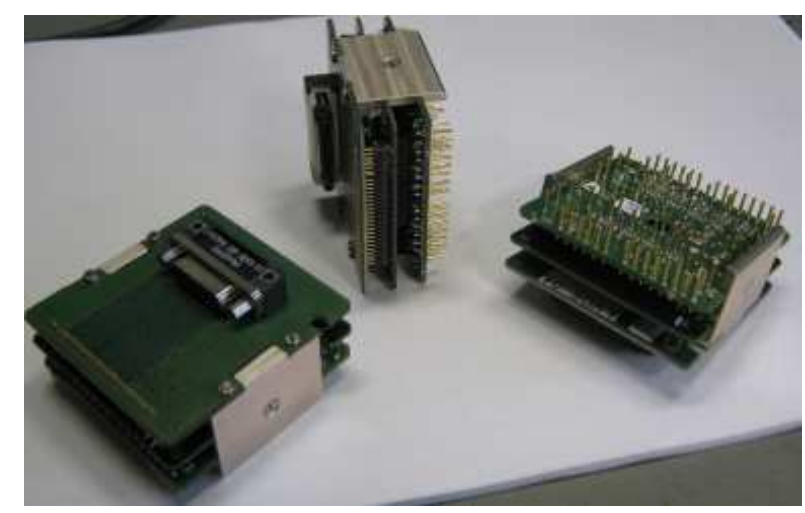

Figure 4. Photographs of assembled Front-End Electronics with LVDS read out.

The first one HVRC module is connected to the Hamamatsu MAPMT. HVRC module provides the high voltage to all dynodes of the MAPMT and picks the signals from the 64 anodes. The signals pass through the $\mathrm{RC}$ element to the next PCB for further processing. It is the ASIC PCB - the main component of all three frontend parts. It preprocesses the analog signals using the GAMMA MEDICA (Ideas) VA64TAP3 ASIC chip and LS64 level shifter. These components provide digital pattern of the signals from all the anodes as well as the sample-and-hold analog buffer for reading of 64 analog amplitudes. Fast trigger logic is formed using dedicated output lines from the ASIC. They are coupled to discrete discriminators to generate full information about the event structure. Two main features event multiplicity and amplitudes are the most important to define the event logic for the trigger. All data processing is controlled by the Actel Igloo AGL250V2, programmable FLASH-based FPGA chip. The role of the last board IF PCB is twofold: to provide means for the bi-directional communication with the Central Trigger utilizing the LVDS communication and assure the proper quality of the power supply voltages. The power consumptions of one POLAR Frontend unit is slightly below $1.2 \mathrm{~W}$ including the power lost by the PMT voltage divider.

\subsubsection{Central Trigger}

The Frontend modules are interconnected with the event acceptance decision. The Central Trigger serves in analysis of the frontend outputs during event detection. Only events with preselected logic can be accepted. During the normal operation the system searches for coincidence signals within a time window of $\approx 100 \mathrm{~ns}$. They should come from at least two plastic bars, either on the level of a single module or the entire system of 25 modules. Such predefined trigger condition is based on the fact that to measure the polarization of X-rays, POLAR instrument detects the interaction using Compton scattering process. The incoming photon must scatter at least twice (and in different bars) in order to define its polarization state. The Central Trigger subsystem utilizes three Actel ProAsic FPGA chips, which are responsible for different working modes: communication FPGA (reads 25 modules), trigger FPGA, central processing FPGA. The firmware is optimized for automatic detection of bursts and, in case of a burst occurrence, it stores the events in a dedicated FLASH memory. The 
on-board memory, together with the presence of dedicated FIFOs blocks at the Frontend FPGAs as well as LVDS communication speed of $20 \mathrm{Mbps}$, ensure that the apparatus is able to sustain a continuous rate of several $\mathrm{kHz}$.

\section{SPACE QUALIFICATION AND PERFORMANCE TESTS}

In order to fill in regulation and requirements of the GESSA agency the set of procedures has been developed and is now implemented for test and qualification of QM and FM units of the POLAR instrument.

\subsection{FEE Tests}

Functionality tests for fully assembled FFE units include: basic quality control tests at the production level (components screening, soldering etc.), electrical tests (voltages, power consumption, protection circuits), functional tests (communication with central unit, power interface, readout, test of analog and digital circuits, trigger). These tests are performed before and after conformal coating and thermal cycling. Calibration of all channels with pulser generator as well as the noise and dark current measurements are carried out during the second stage of the qualification tests.

The purpose of the pulser test system is to provide means for testing, commissioning, and calibration of the gains and timing parameters of all FEE channels. The generator is based on the ADG749 changeover switch. This monolithic CMOS SPDT switch is designed using the submicron process that provides low power dissipation yet gives high switching speed, low on resistance, and low leakage currents. The pulser generates single or periodical voltage pulses with several adjustable parameters: pulse amplitude, repetition rate and time intervals between consecutive pulses. The typical ADC output with pulser scan over a wide range of amplitude is presented in Figure 5.

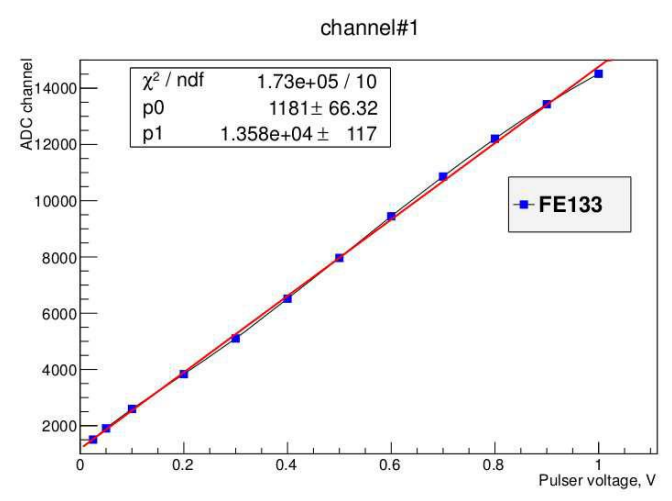

Figure 5. Typical ADC channels response as function of input signals from the pulser generator (arbitrary units)

\subsection{Performance Tests}

To fully validate the functionality and performance of the POLAR detector several modules were illuminated with the high-energy, 100\% polarized X-rays. Two beam test campaigns with synchrotron radiation at ESRF(Grenoble,France) were conducted in December 20097 and November 2011.

Tests with synchrotron radiation confirmed the polarimetric capabilities of POLAR detector, which for the measurements consisted of 4x64 plastic bars arranged as 2x2 array of target-MAPMT-FEE-modules.

The measurements have been made with a broad range of beam energies from $100 \mathrm{keV}$ up to $511 \mathrm{keV}$ and various polarization angles. The results proved excellent polarimetric aptitudes of POLAR. Modulation factors $\mu_{100}$ between $25 \%$ and $50 \%$ were obtained (See Fig. 6 ). In addition, their strong dependence on the beam energy and selection cuts was also confirmed.

Analysis technique that requires the presence of an unpolarized sample of photons (experimental or simulated) provides very good fit results, and a second analysis independent from the simulations and from the presence of 
the unpolarized sample of data was also able to reconstruct the polarization parameters with a good accuracy. Both analyses reconstruct the polarization angle with an accuracy better than $2^{\circ}$.

The tests at ESRF demonstrated that the polarimetric capabilities of POLAR are very good and in agreement with Monte Carlo simulations.



Figure 6. Example of a modulation curve measurement for 100\% polarized synchrotron radiation beam.

The simulation performed with GEANT4 were capable of reproducing the experimental results. This served us also as a validation of the Monte Carlo model that have been already used for several studies related to POLAR in the past years. Therefore one can use the same model upgraded with the model of the Space station for further simulation studies in the future.

Measurements with low-energy synchrotron radiation at the Swiss Light Source (SLS) in PSI (Villigen, Switzerland) have been performed to study the energy region near the trigger threshold for the single hit (5 $\mathrm{keV})$. Data analysis is currently ongoing.

\subsection{Space Qualification Tests}

To guarantee that the POLAR detector will pass the launch intact and ensure its fully operational potential in the severe environmental conditions present in orbit, a series of space qualification tests have been started with the POLAR EQM in the last six months. Qualification tests include: engineering validation tests, vibration, thermal and thermo-vacuum tests. Several irradiation tests of FEE have been performed with proton and heavy ions beam in PSI, Switzerland and Louvain-la-Neuve, Belgium respectively.

\section{CONCLUSIONS AND OUTLOOK}

POLAR is a novel, compact, space-borne Compton polarimeter designed for a precise measurement of hard Xray polarization and optimized for the detection of Gamma-Ray Burst photons in the energy range 50-500 keV. Beam tests with high-energy synchrotron radiation at ESRF in 2009 and 2011 confirmed that the polarimetric capabilities of POLAR are excellent, as predicted by Monte Carlo simulations. Two qualification models of the instrument are nearly fully manufactured and assembled. In parallel, the preparations for manufacturing of the flight model are running. All above tasks are ongoing in view of the launch of the POLAR instruments onboard of the Chinese spacelab Tian-Gong 2 in 2014.

\section{REFERENCES}

[1] R.W. Klebesadel, I. S. and Olson, R., "Observations of gamma-ray bursts of cosmic origin," ApJ. 182, L85 (1973).

[2] Yonetoku, D. et al., "Detection of gamma-ray polarization in prompt emission of grb 100826a," ApJ 743(2), L30 (2011). 
[3] N.Produit et al., "Polar, a compact detector for gamma-ray bursts photon polarization measurements," Nucl.Instr. and Meth. A 550(3), 616-625 (2005).

[4] Eljen Technology, http://www.eljentechnology.com/, EJ-248M Technical Data Sheet.

[5] Garcia, E. S., X-Ray Polarization: RHESSI Results and POLAR Prospects, PhD thesis, Universite de Geneve (2010).

[6] Orsi, S., "Studies on the 3m vikuiti esr reflector for the polar experiment," Tech. Rep. POLAR DPNC SO TN 003 01, University of Geneva (DPNC) Switzerland (2009).

[7] Orsi, S. et al., "Response of the compton polarimeter polar to polarized hard x-rays," Nucl.Instr. and Meth. A 648(1), 139-154 (2011). 